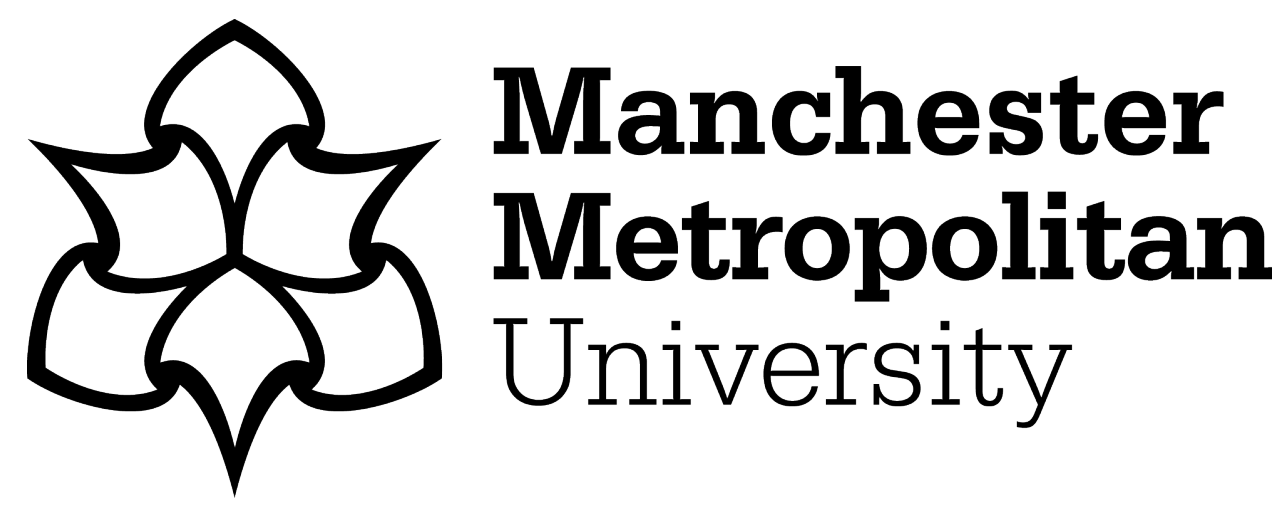

Bestelmeyer, Patricia, Davis, Nicholas, Poliva, Oren and Rafal, Robert (2018) Neuromodulation of Right Auditory Cortex Selectively Increases Activation in Speech-Related Brain Areas in Brainstem Auditory Agnosia. Cognitive and Behavioral Neurology, 31 (3). pp. 151-155. ISSN 0894-878X

Downloaded from: https://e-space.mmu.ac.uk/621541/

Version: Accepted Version

Publisher: Wolters Kluwer Health

DOI: https://doi.org/10.1097/WNN.0000000000000162

Please cite the published version 
Cite as: Bestelmeyer, P. E., Davis, N. J., Poliva, O., \& Rafal, R. D. (2018). Neuromodulation of Right Auditory Cortex Selectively Increases Activation in Speech-Related Brain Areas in Brainstem Auditory Agnosia. Cognitive and Behavioral Neurology, 31(3), 151-155.

\section{Neuromodulation of Right Auditory Cortex Selectively Increases Activation in Speech- Related Brain Areas in Brainstem Auditory Agnosia}

Patricia E.G. Bestelmeyer, PhD, ${ }^{*}$ Nick J. Davis, $\mathrm{PhD}, \dagger$ Oren Poliva, $\mathrm{PhD},{ }^{*}$ and Robert D.

$$
\text { Rafal, MD }
$$

From the *School of Psychology, Bangor University, Bangor, Gwynedd, United Kingdom; $\dagger$ Department of Psychology, Manchester Metropolitan University, Manchester, United Kingdom; and $\ddagger$ Department of Psychological and Brain Sciences, University of Delaware, Newark, Delaware.

[Correspondence: Patricia E.G. Bestelmeyer, PhD, School of Psychology, Bangor University, Gwynedd, LL57 2AS, UK; e-mail: p.bestelmeyer@bangor.ac.uk]

The authors declare no conflicts of interest.

Correspondence: Patricia E.G. Bestelmeyer, PhD, School of Psychology, Bangor University, Gwynedd, LL57 2AS, United Kingdom (e-mail: p.bestelmeyer@bangor.ac.uk).

Running head: Anodal tDCS over Auditory Cortex Modulates Neural Activation in Auditory Agnosia 


\begin{abstract}
Auditory agnosia is an inability to make sense of sound that cannot be explained by deficits in low-level hearing. In view of recent promising results in the area of neurorehabilitation of language disorders after stroke, we examined the effect of transcranial direct current stimulation (tDCS) in a young woman with general auditory agnosia caused by traumatic injury to the left inferior colliculus. Specifically, we studied activations to sound embedded in a block design using functional magnetic resonance imaging before and after application of anodal tDCS to the right auditory cortex. Before tDCS, auditory discrimination deficits were associated with abnormally reduced activations of the auditory cortex and bilateral unresponsiveness of the anterior superior temporal sulci and gyri. This session replicated a previous functional scan with the same paradigm a year before the current experiment. We then applied anodal tDCS over right auditory cortex for 20 minutes and immediately rescanned the patient. We found increased activation of bilateral auditory cortices and, for speech sounds, selectively increased activation in Broca's and Wernicke's areas. Future research might consider the long-term behavioral effects after neurostimulation in auditory agnosia and its potential use in the neurorehabilitation of more general auditory disorders.
\end{abstract}

Key Words: auditory agnosia, inferior colliculus, sound recognition, tDCS

$\mathbf{f M R I}=$ functional magnetic resonance imaging. $\mathbf{t D C S}=$ transcranial direct current stimulation.

Transcranial direct current stimulation (tDCS) is a form of neurostimulation that uses mild constant electrical currents to the brain area of interest via electrodes on the scalp. This 
relatively new brain stimulation technique is used to explore causal relationships between brain regions and cognitive functions, predominantly in healthy individuals.

Neuromodulatory changes induced by tDCS have been observed in a variety of domains, for instance, motor control (Sehm et al, 2013), visual working memory (Heimrath et al, 2012), and language (Iyer et al, 2005). The mechanisms of tDCS are still debated, but it is currently thought that applying anodal tDCS to a particular brain region leads to increased cortical excitability in that region, while applying cathodal tDCS leads to decreased excitability (Nitsche and Paulus, 2000, 2001). Polarity and various additional variables affect brain excitability, including stimulation intensity and duration, cortical neurochemicals, and even genetics (Price et al, 2015).

Transcranial direct current stimulation has been used for therapeutic purposes in various clinical populations because of its ability to induce prolonged brain excitability. The rationale is that if a clinical condition results from altered activity within a particular brain region, then normalizing this activity with tDCS should lead to improvements in that condition (Sale et al, 2015). More specifically, in the field of aphasia rehabilitation after a stroke, the rationale of applying tDCS to recover language is most frequently based on a model of interhemispheric rivalry between the residual speech areas in the damaged left hemisphere and intact right hemisphere (Holland and Crinion, 2012; Otal et al, 2015). According to this model, impaired speech is due to reduced output from the damaged left hemisphere and/or inhibition of the left hemisphere from the intact right hemisphere. Improvements in aphasic patients may therefore be obtained by increasing the excitability in the damaged left hemisphere or dampening the output of the intact hemisphere. In accordance with this model, Monti et al (2008), for example, applied tDCS over the damaged left frontotemporal site in chronic aphasia patients. Performance on a picture naming task was assessed before and after tDCS, with cathodal stimulation enhancing accuracy on the task by 
over $30 \%$. The authors concluded that the improvement could be attributed to disinhibition of the damaged cortex, in line with the model of interhemispheric rivalry.

In contrast, others have obtained performance improvements with anodal tDCS to the contralesional hemisphere. The rationale here is boosting a mechanism in the right hemisphere for language recovery after stroke. For example, long-term treatment success for anomia is best predicted by activity increases in the right-sided Wernicke's homologue (Menke et al, 2009). In addition, the right hemisphere seems to play an important role in recovery from poststroke aphasia whereby right temporal activation has been linked to auditory sentence comprehension (Crinion and Price, 2005). For this reason, Floel et al (2011) applied anodal, cathodal, and sham tDCS over the right temporoparietal cortex in patients with chronic aphasia, but only anodal stimulation resulted in a significant and consistent improvement during language training. In the field of language rehabilitation, tDCS studies are heterogeneous with respect to stimulation site, polarity, duration, and task (Monti et al, 2013).

More recently, tDCS has been used in the rehabilitation of a patient with more general auditory problems after suffering from brainstem encephalitis during childhood (Mori et al, 2016). This patient suffered from bilateral hearing impairment, which was worse in her right ear. Anodal tDCS was applied to her left auditory cortex, resulting in improved speech discrimination performance after only one application. This finding suggests that other neurologic problems resulting in more general auditory perception disabilities may benefit from anodal tDCS, thus supporting neurorehabilitation efforts.

Here we report the effects of anodal tDCS over right auditory cortex on brain activity in a patient with auditory brainstem agnosia, whose case we reported earlier (Poliva et al, 2015). In brief, this young woman suffered damage to her left inferior colliculus and its brachium. The lesion led to partial deafferentation of her auditory cortex, particularly on the 
left side, resulting in partial interruption of her auditory pathways and thereby causing a general auditory agnosia. A functional scan of her brain response to different sound categories (eg, speech, environmental sounds) revealed activations of bilateral caudal subareas of her primary auditory cortex (designated as hA1 in humans) and right posterior superior temporal gyrus. However, the rostral subarea of her primary auditory cortex (designated as $\mathrm{hR}$ in humans) and anterior superior temporal sulci and gyri were unresponsive compared with control participants. Thus, while her auditory ventral stream seems dysfunctional, her residual auditory ability is supported by a largely preserved auditory dorsal stream.

In view of the promising rehabilitation results for language and more general auditory disorders as well as the enhancement in performance of more basic auditory abilities with tDCS (Ladeira et al, 2011), our aim was to explore whether tDCS would benefit this particular patient in recovering some activity in bilateral auditory cortices and association areas. We decided, in the first instance, to recommend a trial of anodal stimulation to the less affected, contralesional hemisphere. The rationale was based on the findings that, for poststroke aphasic patients, long-term training success for naming objects was best predicted by increased activation in right-sided Wernicke's homologue (Menke et al, 2009). Also, this approach aimed to increase reactivity to sound in the less affected, contralesional auditory cortex.

\section{CASE REPORT}

\section{Early Evaluation}

A healthy young woman, M.L., sustained a closed head injury at age 17. At that time, a computed tomography scan revealed a hemorrhage in the right basal ganglia and dorsal midbrain. At 28 years old, her chief disability is a severe auditory agnosia for speech and 
environmental sounds resulting from avulsion of the left inferior colliculus. She has some motor impairment due to diffuse axonal injury but is functionally independent. We have previously reported her medical history, neurologic examinations, neuroimaging, and detailed auditory assessments (Poliva et al, 2015). Patient M.L. was under the neurologic care of author R.D.R. from the time of the injury until his retirement.

Magnetic resonance imaging scans, performed once when the patient was 27 (Poliva et al, 2015) and twice a year later for the current report, revealed a cystic cavity in the right putamen at the site of the previous hemorrhage. It showed a small periventricular lesion on the right lower pons, in the region of the inferior cerebellar peduncle. There is nearly complete avulsion of the left inferior colliculus, sparing only its most medial and caudal parts, and destruction of the brachia of the superior and inferior colliculi with the lesion extending into the red nucleus encroaching on the medial border of the left medial geniculate nucleus. The lesion has led to partial deafferentation of the auditory cortex, particularly on the left side.

While her responses during pure tone audiometry were inconsistent, otoscopy, tympanometry, and otoacoustic emissions were normal in both ears, suggesting normal middle and inner ear function. Auditory brainstem response testing performed at $85 \mathrm{~dB} 8$ months after her injury revealed normal wave I and III latencies in both ears. However, wave V was absent after right ear stimulation and delayed after left ear stimulation, in line with the imaging evidence of a lesion in the inferior colliculus. Probabilistic tractography confirmed that M.L. had preserved bilateral thalamic connectivity to the auditory cortex by showing that her functional anisotropy values were similar to those of a healthy control group (see Poliva et al, 2015, for details).

According to behavioral tests, M.L. suffered from auditory extinction as assessed with a dichotic listening task, an impaired ability to localize sound, and poor auditory temporal 
resolution (Poliva et al, 2015). However, while M.L.'s recognition of sound was poor, her performance on identifying spoken words and environmental sounds improved significantly (from $13 \%$ to $73 \%$ correct) with a four-alternative forced-choice design, suggesting a partially preserved ability to perceive sounds.

\section{Therapeutic Trial of tDCS}

Assessment of auditory agnosia and neuroimaging were conducted under a research protocol approved by the ethics committees of Bangor University and the National Health Service (of the United Kingdom). Options for a therapeutic trial of tDCS, intended to potentially benefit this single patient, were explained to her and her parents by her treating neurologist. Although the patient had severe hearing impairment, her ability to read was preserved. The discussion of therapeutic options was conducted in writing and recorded in the patient's clinical record. We obtained the patient's written informed consent to participate and for us to publish her fully anonymized data.

The patient was told that despite some clinical research trials of tDCS for treatment of other neurologic problems, her condition was rare and no clinical trials of tDCS were currently available to her. She was also told about promising trials of tDCS in the treatment of neurologic patients with impaired language comprehension, with no adverse effects of tDCS having been reported in these studies.

The procedure for neurostimulation was explained, as was the sensation she might experience. The basic principle of altering cortical excitability was explained as follows. Weak electrical stimulation of auditory cortex can make brain cells more or less likely to respond to sounds. The analogy given was that adding or removing water from a glass can make it more or less likely to overflow if a pebble is put in the glass. 
R.D.R. explained that the auditory cortex on both sides of her brain had not been damaged, but had been made less responsive to sounds. Also, she was told that we could stimulate either the right or left side of her brain with currents that would cause an increase or decrease of responsiveness. In the first instance, it was decided to recommend anodal stimulation on the right based on observations that right temporal activation predicts auditory sentence comprehension in poststroke aphasic patients (Crinion and Price, 2005).

Before recommending therapeutic, daily stimulation (accompanying behavioral training) for 1 or 2 weeks, we suggested that she have a single session of right anodal temporal lobe stimulation to assess her tolerance. We also aimed to test whether stimulation increased the reactivity of the underlying cortex (using fMRI) or improved her temporal resolution (using the two-click paradigm, described in Poliva et al, 2015). The plan outlined to the patient was that, if tDCS was well tolerated and caused no adverse effects, and if there was either an increase in right temporal blood flow or an improvement in auditory temporal resolution, she would be offered a course of tDCS treatment.

\section{METHODS}

\section{fMRI Paradigm, Acquisition, and Analysis}

We scanned M.L. (age = 28 years) before and after tDCS on a block design based on the "voice localizer" (Belin et al, 2000), which we modified to include blocks of speech sounds. It consisted of four sound categories, each presented as twenty 10 -second blocks, ie, vocal nonspeech sounds (eg, laughs, coughs), verbal sounds (different words), environmental sounds (eg, car engine, doorbell), and silent baseline. Sounds were presented via headphones (NordicNeuroLab, Bergen, Norway) at 85 decibels of sound pressure level (C-weighting) and superimposed on scanner noise. Each block started with 2 seconds of silence followed by 8 seconds of different stimuli of the same category. The 80 blocks were presented 
pseudorandomly (ie, no presentation of two same-category blocks in succession). We obtained a structural scan in each MRI session. The first MRI scans, tDCS, and the second MRI scans were carried out within approximately 1 hour.

Scans were performed on a 3T Philips Achieva (Eindhoven, the Netherlands) with an 8-channel head coil. The T2*-weighted scan consisted of an echo-planar imaging sequence in interleaved ascending order (32 axial slices, voxel size: $3 \mathrm{~mm}^{3}$; flip angle: 90 degrees; field of view $=240 ;$ repetition time $=2$ seconds; echo time $=30 \mathrm{msec} ; 400$ volumes plus 20 volumes of additional rest at the end; 14 minutes). We then performed a whole-brain T1-weighted scan (voxel size: $1 \mathrm{~mm}^{3}$; flip angle: 8 degrees; field of view $=240$; repetition time $=12 \mathrm{msec}$; echo time $=3.5 \mathrm{msec} ; 5$ minutes $)$.

Data were analyzed in native space using SPM8 (Friston et al, 2006). Preprocessing consisted of anterior commissure-posterior commissure alignment, corrections for head motion (spatial realignment; trilinear interpolation), coregistration, and spatial smoothing by applying a Gaussian kernel of $6 \mathrm{~mm}$ full width at half maximum. Our SPM matrix contained three regressors of interest (voice, speech, and environmental sounds) and movement parameters as regressors of no interest for each tDCS session. We calculated t-contrasts for pre- versus post-tDCS sessions (ie, areas that responded more or less after tDCS across all sound categories and specifically in each category). Results are from a whole-brain analysis and reported at an extent threshold of $\mathrm{k}>35$ voxels and familywise error-corrected at the cluster level with a threshold of $P<0.05$.

\section{tDCS Delivery}

Following the first MRI session, we delivered tDCS using a Magstim DC-Plus stimulator (Whitland, United Kingdom), using saline-soaked sponge-covered rubber electrodes. Electrode positions were determined using the 10-20 international electrode 
placement system. The anode $(5 \times 5 \mathrm{~cm})$ was placed at position $\mathrm{T} 4$, which overlies the right temporal lobe (Koessler et al, 2009), and the cathode $(5 \times 7 \mathrm{~cm})$ was placed horizontally over the contralateral orbit. The COMETS toolbox (Jung et al, 2013) was used to model the electrical field resulting from this montage over a "standard" brain and showed the concentration of energy in the intended location of auditory cortex. A current of $1.5 \mathrm{~mA}$ was delivered for 20 minutes, during which M.L. performed no experimental task but her cutaneous sensations were monitored.

\section{RESULTS}

Auditory temporal resolution was measured immediately before tDCS and immediately after (ie, before the patient was placed in the MRI scanner). The task was the same brief two-click fusion test used to test the patient previously (Poliva et al, 2015), and took less than 2 minutes to administer. (A very brief behavioral test was necessary because the effects of tDCS last about 20 minutes, and it was critical that scanning be completed during this time frame.) Auditory temporal resolution was unchanged after tDCS, and did not differ from the previous testing reported by Poliva et al (2015).

Before tDCS, the patient's primary auditory cortex field (hA1) was active, but the rostral subarea of her primary auditory cortex $(\mathrm{hR})$ and anterior superior temporal gyri and sulci were unresponsive to sound at a threshold of $P<0.001$ (uncorrected; $\mathrm{k}=0$ ), replicating a previous scan 1 year earlier with the same paradigm (Poliva et al, 2015).

Contrasting the two fMRI sessions that were run within an hour before and after the application of tDCS, we found greater blood oxygenation level-dependent activation in bilateral auditory cortices to all sounds against a silent baseline after anodal tDCS was applied (left: $\mathrm{k}=224, \mathrm{~T}=9.95, P<0.001$; right: $\mathrm{k}=348, \mathrm{~T}=7.22, P<0.001)$ compared with before tDCS (left: $\mathrm{k}=82, \mathrm{~T}=4.95, P<0.001$; right: $\mathrm{k}=156, \mathrm{~T}=8.48, P<0.001$; 
Figure 1A). We also saw a selective increase in activation to speech after tDCS (speech pretDCS $<$ speech post-tDCS; Figure 1B) in the left inferior frontal gyrus $(\mathrm{k}=66, \mathrm{~T}=4.44, P<$ 0.001; Broca's area) and posterior superior temporal sulcus $(\mathrm{k}=86, \mathrm{~T}=4.95, P<0.001$; Wernicke's area). We found no specific effects of tDCS to environmental and vocal sound categories and no increased activations for any of the reverse contrasts (ie, post-tDCS $<$ pretDCS).

[insert Figure 1 about here]

\section{DISCUSSION}

In light of encouraging reports in the language rehabilitation literature, we examined whether a patient with brainstem auditory agnosia might benefit from anodal tDCS to the less affected hemisphere. We scanned our patient before and after anodal tDCS over the right auditory cortex while she passively listened to different sound categories, including speech. Before the application of tDCS, much of auditory cortex was unresponsive bilaterally independent of sound category, which is in line with patient M.L.'s inability to reliably recognize and discriminate sound objects.

After the application of tDCS, blood oxygenation level-dependent activation increased along primary auditory cortices medially and along the superior temporal gyri and sulci. We also found selective activation to speech sounds in Broca's and Wernicke's areas when there was no selective activation to any of the sound categories before the application of tDCS. Our results highlight the potential of tDCS to selectively increase blood flow, but it remains to be seen what this increased brain response means in terms of function.

Importantly, an increase in MRI activation after tDCS is unlikely to be due to a repetition in testing. This is because the test-retest reliability of activation in the superior 
temporal sulcus for the voice localizer is high (intraclass correlation coefficient $>0.9$ ) (Pernet et al, 2015). Also noteworthy is that the pattern of activation is not due to differences in head motion between the two MRI sessions, as patient M.L. was instructed to stay as still as possible and movement parameters were included in the models. Head motion was also within $2.5 \mathrm{~mm}$ along $x$-, $y$-, and $z$-axes in both sessions (ie, comparable to scanning sessions of young, healthy individuals).

Patient M.L.'s performance on a very brief two-click fusion test remained unchanged after the administration of tDCS. While there were practical reasons why we did not examine the patient on a more sensitive and extensive test battery during this pilot administration of tDCS, it makes it difficult to speculate what the increased activation in auditory cortex, and in particular the enhanced differential activation to speech, may reflect. Jäncke et al (1998) have shown that increased sound intensity (ie, sound pressure level) results in increased fMRI response in bilateral primary and secondary auditory cortices and bilateral inferior frontal gyri to verbal and nonverbal stimuli. Applying tDCS to the right auditory cortex may have affected sound intensity perception by triggering increased efferent corticofugal activity and thereby affected auditory cortical processing. This explanation, however, cannot explain the differential activation to sounds of the speech category compared with other sound types. Future studies, initially with healthy participants, will need to carefully investigate what this increased fMRI response after tDCS to speech may reflect, ideally using a psychophysics approach (eg, Bestelmeyer et al, 2011).

We originally collected these data as a pilot for a therapeutic trial of tDCS over the course of a week. Before starting such a trial, we wanted to ensure that the patient tolerated tDCS well. On the day of testing, M.L. reported no adverse effects of tDCS. However, on a follow-up visit a week later, she mentioned that her ability to understand speech seemed worse for a couple of days after the day of her testing. This subjective report of delayed 
worsening of auditory perception is difficult to interpret and may be the result of a number of different factors not necessarily related to our testing. We are not aware of similar reports in the literature describing a delayed worsening in performance. However, given this report we decided not to proceed with any further brain stimulation of this patient and exploration of tDCS-induced behavioral effects.

Our findings are in line with models describing dual pathways in the auditory system known as the dorsal and ventral processing streams for the analysis of spatial location and object (including speech) processing, respectively (Bizley and Cohen, 2013). This patient's auditory agnosia seems to be caused by damage to the auditory ventral stream. Application of tDCS led to partial "normalization" of brain activity in this pathway (ie, the primary auditory cortices and anterior superior temporal sulci and gyri). Future research might investigate whether this "normalization" following tDCS can be linked to enhanced behavioral performance as well as documenting the time course of performance over several weeks.

\section{ACKNOWLEDGMENTS}

We are very grateful to M.L. for her continued patience and participation in our studies.

\section{REFERENCES}

Belin P, Zatorre RJ, Lafaille P, et al. 2000. Voice-selective areas in human auditory cortex. Nature. 403:309-312.

Bestelmeyer PEG, Belin P, Grosbras MH. 2011. Right temporal TMS impairs voice detection. Curr Biol. 21:R838-839.

Bizley JK, Cohen YE. 2013. The what, where and how of auditory-object perception. Nat Rev Neurosci. 14:693-707. 
Crinion J, Price CJ. 2005. Right anterior superior temporal activation predicts auditory sentence comprehension following aphasic stroke. Brain. 128:2858-2871.

Floel A, Meinzer M, Kirstein R, et al. 2011. Short-term anomia training and electrical brain stimulation. Stroke. 42:2065-2067.

Friston KJ, Ashburner JT, Kiebel SJ, et al. 2006. Statistical Parametric Mapping: The Analysis of Functional Brain Images. London, United Kingdom: Elsevier.

Heimrath K, Sandmann P, Becke A, et al. 2012. Behavioral and electrophysiological effects of transcranial direct current stimulation of the parietal cortex in a visuo-spatial working memory task. Front Psychiatry. 3:56.

Holland R, Crinion J. 2012. Can tDCS enhance treatment of aphasia after stroke? Aphasiology. 26:1169-1191.

Iyer MB, Mattu U, Grafman J, et al. 2005. Safety and cognitive effect of frontal DC brain polarization in healthy individuals. Neurology. 64:872-875.

Jäncke L, Shah NJ, Posse S, et al. 1998. Intensity coding of auditory stimuli: an fMRI study. Neuropsychologia. 36:875-883.

Jung YJ, Kim JH, Im CH. 2013. COMETS: a MATLAB toolbox for simulating local electric fields generated by transcranial direct current stimulation (tDCS). Biomed Eng Lett. $3: 39-46$.

Koessler L, Maillard L, Benhadid A, et al. 2009. Automated cortical projection of EEG sensors: anatomical correlation via the international 10-10 system. Neuroimage. $46: 64-72$.

Ladeira A, Fregni F, Campanha C, et al. 2011. Polarity-dependent transcranial direct current stimulation effects on central auditory processing. PLoS One. 6(9):e25399.

Menke R, Meinzer M, Kugel H, et al. 2009. Imaging short- and long-term training success in chronic aphasia. BMC Neurosci. 10:1-13. 
Monti A, Cogiamanian F, Marceglia S, et al. 2008. Improved naming after transcranial direct current stimulation in aphasia. J Neurol Neurosurg Psychiatry. 79:451-453.

Monti A, Ferrucci R, Fumagalli M, et al. 2013. Transcranial direct current stimulation (tDCS) and language. J Neurol Neurosurg Psychiatry. 84:832-842.

Mori T, Takeuchi N, Suzuki S, et al. 2016. Anodal transcranial direct current stimulation over the auditory cortex improved hearing impairment in a patient with brainstem encephalitis. J Int Med Res. 44:760-764.

Nitsche MA, Paulus W. 2000. Excitability changes induced in the human motor cortex by weak transcranial direct current stimulation. J Physiol. 527:633-639.

Nitsche MA, Paulus W. 2001. Sustained excitability elevations induced by transcranial DC motor cortex stimulation in humans. Neurology. 57:1899-1901.

Otal B, Olma MC, Floel A, et al. 2015. Inhibitory non-invasive brain stimulation to homologous language regions as an adjunct to speech and language therapy in poststroke aphasia: a meta-analysis. Front Hum Neurosci. 9:236.

Pernet CR, McAleer P, Latinus M, et al. 2015. The human voice areas: spatial organization and inter-individual variability in temporal and extra-temporal cortices. Neuroimage. 119:164-174.

Poliva O, Bestelmeyer PEG, Hall M, et al. 2015. Functional mapping of the human auditory cortex: fMRI investigation of a patient with auditory agnosia from trauma to the inferior colliculus. Cogn Behav Neurol. 28:160-180.

Price AR, McAdams H, Grossman M, et al. 2015. A meta-analysis of transcranial direct current stimulation studies examining the reliability of effects on language measures. Brain Stimul. 8:1093-1100. 
Sale MV, Mattingley JB, Zalesky A, et al. 2015. Imaging human brain networks to improve the clinical efficacy of non-invasive brain stimulation. Neurosci Biobehav Rev. $57: 187-198$.

Sehm B, Kipping J, Schafer A, et al. 2013. A comparison between uni- and bilateral tDCS effects on functional connectivity of the human motor cortex. Front Hum Neurosci. $7: 183$ 
Figure Legend

\section{(A) All sounds}
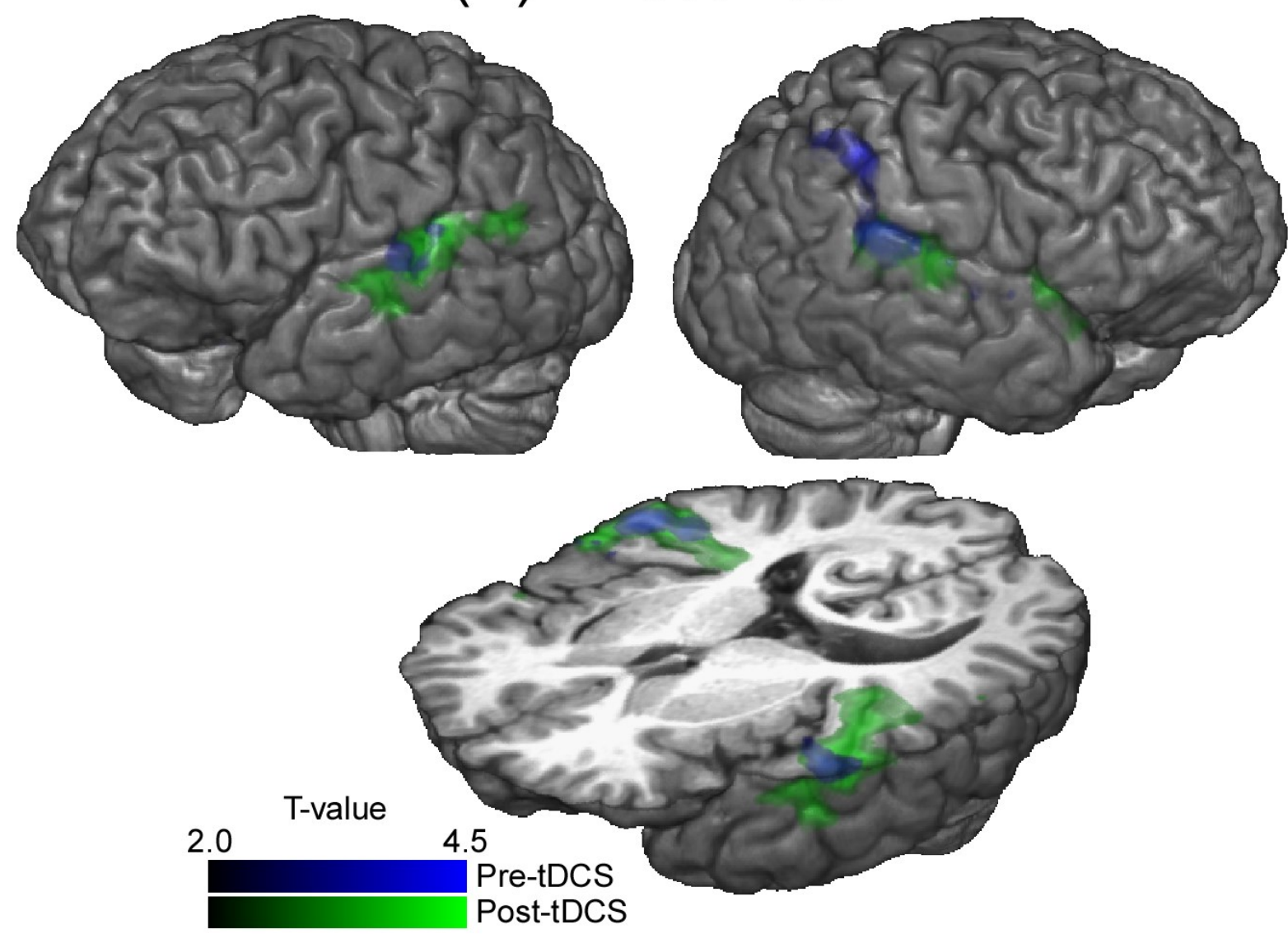

\section{(B) Speech}

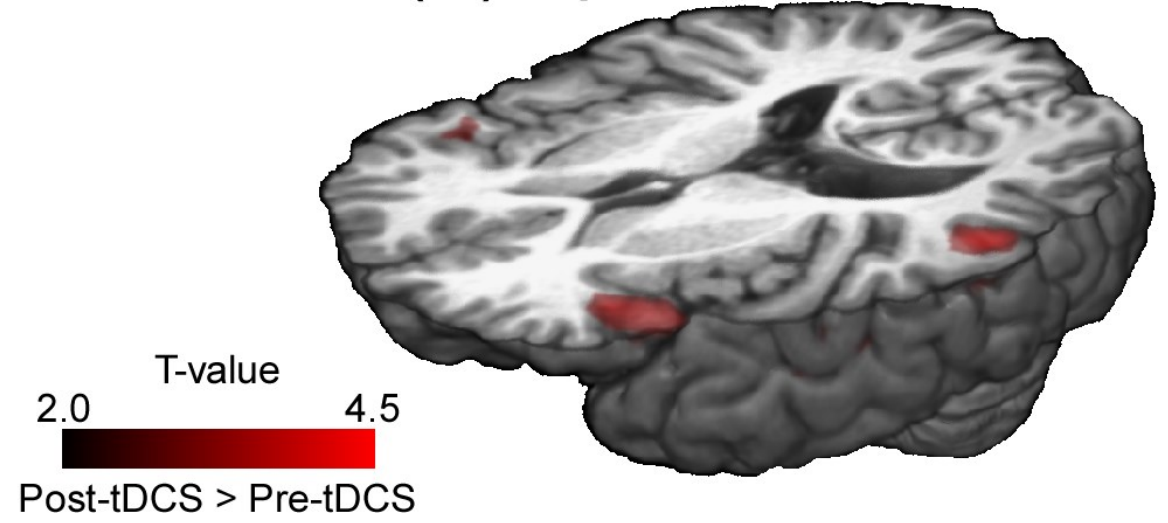

FIGURE 1. Illustration of brain activation in the patient. A: Activation to any type of sound compared to silence pre-tDCS and post-tDCS . B: Illustration of the contrast of activation in response to speech sounds post-tDCS $>$ pre-tDCS. 